\title{
A fractional order HIV/AIDS epidemic model with Mittag-Leffler kernel
}

\author{
Muhammad Aslam¹, Rashid Murtaza' ${ }^{1}$ Thabet Abdeljawad ${ }^{2,3,4^{*}}$, Ghaus ur Rahman , Aziz Khan², \\ Hasib Khan ${ }^{6 *}$ and Haseena Gulzar ${ }^{7}$
}

\author{
"Correspondence: \\ tabdeljawad@psu.edu.sa; \\ hasibkhan13@yahoo.com \\ ${ }^{2}$ Department of Mathematics and \\ General Sciences, Prince Sultan \\ University, Riyadh, Saudi Arabia \\ ${ }^{6}$ Department of Mathematics, \\ Shaheed Benazir Bhutto University, \\ Sheringal, Dir Upper, Khyber \\ Pakhtunkhwa, Pakistan \\ Full list of author information is \\ available at the end of the article
}

\begin{abstract}
In this article, we study a fractional order HIV/AIDS infection model with ABC-fractional derivative. The model is based on four classes of a population. The study includes the existence and uniqueness of solution, the stability analysis, and simulations. We utilize the fixed point technique for the existence and uniqueness analysis. The stability of the fractional order model is derived with the help of existing literature for the Hyers-Ulam stability. For the numerical computations, the Lagrange interpolation is utilized, and the simulations are obtained for specific parameters. The results are closer to the classical results for different orders.
\end{abstract}

Keywords: Fractional order HIV/AIDS model; Existence of solution; Hyers-Ulam stability; Numerical solution

\section{Introduction}

Infectious diseases have been documented as an unremitting risk to human beings. Transmissible diseases are those which transfer from animals to humans or from humans to humans. The spread of these diseases occurs through different sources, including airborne viruses, bacteria, and body fluids like blood, urine, spit, breast milk, tears, and many more. Among the transmissible diseases, acquired immunodeficiency syndrome (AIDS) is a transferable disease, and human immunodeficiency virus (HIV) is the causative source for AIDS which weakens the role of the body to fight against diseases and leaves it open to attack of usually safe infections. HIV targets CD4+ T-cells and replicates rapidly. In the initial stage of infection, the plasma holding high level of HIV virus particles covers the whole body and is present in both free virus particles as well the virus within infected immune cells. Because of the important role of CD4+ T-cells in immune regulation, their reduction and destruction causes decrease in the ability of the immune system to fight. The decrease in these cells is used in a clinical examination as pointer for AIDS (see [1-4] for more details). Recently, scientists have presented several models for the human immune system, and a large number of articles can be studied in the area of HIV infection of CD4+ T-cells to understand HIV infection, HIV dynamics, disease progression, and interaction of the immune system with HIV. In this area, the primary model describing the HIV infection was developed by Perelson [2], which was later on modified by Perelson et al. [3].

(c) The Author(s) 2021. This article is licensed under a Creative Commons Attribution 4.0 International License, which permits use sharing, adaptation, distribution and reproduction in any medium or format, as long as you give appropriate credit to the original author(s) and the source, provide a link to the Creative Commons licence, and indicate if changes were made. The images or other third party material in this article are included in the article's Creative Commons licence, unless indicated otherwise in a credit line to the material. If material is not included in the article's Creative Commons licence and your intended use is not permitted by statutory regulation or exceeds the permitted use, you will need to obtain permission directly from the copyright holder. To view a copy of this licence, visit http://creativecommons.org/licenses/by/4.0/. 
They studied that the model shows many of the symptoms of AIDS which were clinically analyzed such as the decrease of $\mathrm{CD} 4+\mathrm{T}$-cells, the long latency period, low levels of free virus in the body, and many more.

Recently, some researchers have developed very impressive articles for the mathematical description of the HIV/AIDS models. Among those, Mukandavire et al. [5] presented a mathematical model for the HIV/AIDS spread on sex-base as a delay-system of differential equations and gave the local and global stabilities for their model subjected to the value of basic reproduction number $R_{0}$. Tabassum et al. [6] developed a nonlinear mathematical model for the HIV/AIDS transmission and examined the necessary conditions required for the well-posedness and boundedness. Dutta and Gupta [7] presented a mathematical model for HIV/AIDS analysis with weak CD4+ T cells and studied the infection, infectionfree equilibrium situations.

The modeling in the fractional order has got more valuable attention of scientists due to diverse analysis of dynamical problems. One can see the use of different mathematical techniques for handling these models. For instance, Nazir et al. [8] used the CaputoFabrizio derivative sense of fractional order derivative for the study of HIV model. They produced existence, stability, and numerical simulations in their work. Sweilam et al. [9] analyzed a variable order fractional co-infection optimal control model of HIV vs malaria. They described the necessary assumptions for the control of the spread in their study. One can see some more related fractional order models and their analysis in the recent developments in [10-15] and many more in the literature.

Keeping in view the importance of mathematical modeling and the use of fractional order operators, we consider the following HIV/AIDS model for the existence, stability, and numerical simulations using the Atangana-Baleanu fractional derivative in the Caputo sense

$$
\left\{\begin{aligned}
&{ }_{0}^{A B C} \mathcal{D}_{t}^{\alpha_{1}} \mathcal{S}(t)= \pi-\beta\left(\mathcal{I}(t)-\eta_{c} \mathcal{C}(t)+\eta_{A} \mathcal{A}(t)\right) \mathcal{S}(t)-\mu_{0} \mathcal{S}(t), \\
&{ }_{0}^{A B C} \mathcal{D}_{t}^{\alpha_{1}} \mathcal{I}(t)= \beta\left(\mathcal{I}(t)-\eta_{c} \mathcal{C}(t)+\eta_{A} \mathcal{A}(t)\right) \mathcal{S}(t)-\left(\rho+\phi+\mu_{0}\right) \mathcal{I}(t) \\
&+\omega \mathcal{C}(t)+\alpha \mathcal{A}(t), \\
&{ }_{0}^{A B C} \mathcal{D}_{t}^{\alpha_{1}} \mathcal{C}(t)= \phi \mathcal{I}(t)-\left(\omega+\mu_{0}\right) \mathcal{C}(t), \\
&{ }_{0}^{A B C} \mathcal{D}_{t}^{\alpha_{1}} \mathcal{A}(t)=\rho \mathcal{I}(t)-\left(\alpha+\mu_{0}+d\right) \mathcal{A}(t),
\end{aligned}\right.
$$

where the total population is $\mathcal{S}(t), \mathcal{I}(t), \mathcal{C}(t), \mathcal{A}(t)$. For this model, $\{\mathcal{S}(t), \mathcal{I}(t), \mathcal{C}(t), \mathcal{A}(t)\}$ represents the classes as follows: $(\mathcal{S})$ the exposed people with HIV, $(\mathcal{I})$ the contaminated/infected, $(\mathcal{C})$ anti-viral treatment (ART) underneath cure, $\mathcal{A}(t)$ is the class of people with AIDS. Here, $\beta$ is the joining rate for HIV diffusion, $\eta_{A} \geq 1$ is a drug parameter, for irresistibleness of a patient with AIDS side effects, while $\eta_{C} \geq 1$ is for the halfway repair of the immune capacity of HIV patients who are treated under ART cure. The model is considered in the $\mathrm{ABC}$ sense of fractional derivative. For details, the readers can benefit from [16-20].

About the ABC-fractional calculus, we highlight the following useful literature from [21-25]. 
Definition 1.1 The ABC-fractional differential operator on $\psi \in H^{*}(a, b), b>a$, for $\alpha_{1} \in$ $[0,1]$ is

$$
{ }_{a}^{A B C} \mathcal{D}_{\tau}^{\alpha_{1}} \psi(\tau)=\frac{B\left(\alpha_{1}\right)}{1-\alpha_{1}} \int_{a}^{\tau} \psi^{\prime}(s) E_{\alpha_{1}}\left[\frac{-\alpha_{1}(\tau-s)^{\alpha_{1}}}{1-\alpha_{1}}\right] d s,
$$

where $B\left(\alpha_{1}\right)$ satisfies the property $B(0)=B(1)=1$.

Definition 1.2 For $\psi \in H^{*}(a, b), b>a, \alpha_{1} \in[0,1]$, the ABR-fractional derivative is

$$
{ }_{a}^{A B R} \mathcal{D}_{\tau}^{\alpha_{1}} \psi(\tau)=\frac{B\left(\alpha_{1}\right)}{1-\alpha_{1}} \frac{d}{d \tau} \int_{a}^{\tau} \psi(s) E_{\alpha_{1}}\left[\frac{-\alpha_{1}(\tau-s)^{\alpha_{1}}}{1-\alpha_{1}}\right] d s .
$$

Definition 1.3 The AB-integral of $\psi \in H^{*}(a, b), b>a, 0<\alpha_{1}<1$ is given by

$$
{ }_{a}^{A B} \mathcal{I}_{\tau}^{\alpha_{1}} \psi(\tau)=\frac{1-\alpha_{1}}{B\left(\alpha_{1}\right)} \psi(\tau)+\frac{\alpha_{1}}{B\left(\alpha_{1}\right) \Gamma\left(\alpha_{1}\right)} \int_{a}^{\tau} \psi(s)(\tau-s)^{\alpha_{1}-1} d s .
$$

Lemma 1.4 The AB-fractional derivative and AB-fractional integral of the function $\psi$ satisfy the Newton-Leibniz formula

$$
\left.{ }_{a}^{A B} \mathcal{I}_{\tau}^{\alpha_{1}}{ }_{a}^{A B C} \mathcal{D}_{\tau}^{\alpha_{1}} \psi(\tau)\right)=\psi(\tau)-\psi(a) .
$$

\section{Existence criteria}

By the AB-fractional integral and HIV/AIDS model (1), we have

$$
\begin{aligned}
& \mathcal{S}(t)-\mathcal{S}(0) \\
&= \frac{1-\alpha_{1}}{\beta\left(\alpha_{1}\right)}\left(\pi-\beta\left(\mathcal{I}(t)-\eta_{c} \mathcal{C}(t)+\eta_{A}, \mathcal{A}(t)\right) \mathcal{S}(t)-\mu_{0} \mathcal{S}(t)\right) \\
&+\frac{\alpha_{1}}{\beta\left(\alpha_{1}\right) \Gamma\left(\alpha_{1}\right)} \int_{0}^{t}(t-s)^{\alpha_{1}-1}\left(\pi-\beta\left(\mathcal{I}(t)-\eta_{c} \mathcal{C}(t)+\eta_{A} \mathcal{A}(t)\right)\right. \\
&\left.\times \mathcal{S}(t)-\mu_{0} \mathcal{S}(t)\right) d s, \\
& \mathcal{I}(t)- \mathcal{I}(0) \\
&=\frac{1-\alpha_{1}}{\beta\left(\alpha_{1}\right)}\left(\beta\left(\mathcal{I}(t)-\eta_{c} \mathcal{C}(t)+\eta_{A} \mathcal{A}(t)\right) \mathcal{S}(t)-\left(\rho+\phi+\mu_{0}\right) \mathcal{I}(t)+\omega \mathcal{C}(t)\right. \\
&+\alpha \mathcal{A}(t))+\frac{\alpha_{1}}{\beta\left(\alpha_{1}\right) \Gamma\left(\alpha_{1}\right)} \int_{0}^{t}(t-s)^{\alpha_{1}-1}\left(\beta\left(\mathcal{I}(t)-\eta_{c} \mathcal{C}(t)+\eta_{A} \mathcal{A}(t)\right) \mathcal{S}(t)\right. \\
&\left.-\left(\rho+\phi+\mu_{0}\right) \mathcal{I}(t)+\omega \mathcal{C}(t)+\alpha \mathcal{A}(t)\right) d s, \\
& \mathcal{C}(t)- \mathcal{C}(0) \\
&= \frac{1-\alpha_{1}}{\beta\left(\alpha_{1}\right)}\left(\phi \mathcal{I}(t)-\left(\omega+\mu_{0}\right) \mathcal{C}(t)+\frac{\alpha_{1}}{\beta\left(\alpha_{1}\right) \Gamma\left(\alpha_{1}\right)} \int_{0}^{t}(t-s)^{\alpha_{1}-1}\right. \\
& \times\left(\phi \mathcal{I}(t)-\left(\omega+\mu_{0}\right) \mathcal{C}(t)\right) d s, \\
& \mathcal{A}(t)-\mathcal{A}(0) \\
&= \frac{1-\alpha_{1}}{\beta\left(\alpha_{1}\right)}\left(\rho \mathcal{I}(t)-\left(\alpha+\mu_{0}+d\right) \mathcal{A}(t)\right)+\frac{\alpha_{1}}{\beta\left(\alpha_{1}\right) \Gamma\left(\alpha_{1}\right)} \int_{0}^{t}(t-s)^{\alpha_{1}-1}
\end{aligned}
$$




$$
\times\left(\rho \mathcal{I}(t)-\left(\alpha+\mu_{0}+d\right) \mathcal{A}(t)\right) d s
$$

Assume that the functions $\mathcal{Q}_{i}$ for $i=1,2,3,4$ are given as follows:

$$
\begin{aligned}
& \mathcal{Q}_{1}(t, S)=\pi-\beta\left(\mathcal{I}(t)-\eta_{c} \mathcal{C}(t)+\eta_{A} \mathcal{A}(t)\right) \mathcal{S}(t)-\mu_{0} \mathcal{S}(t) \\
& \mathcal{Q}_{2}(t, \mathcal{I})=\beta\left(\mathcal{I}(t)-\eta_{c} \mathcal{C}(t)+\eta_{A} \mathcal{A}(t)\right) \mathcal{S}(t)-\left(\rho+\phi+\mu_{0}\right) \mathcal{I}(t)+\omega \mathcal{C}(t)+\alpha \mathcal{A}(t), \\
& \mathcal{Q}_{3}(t, \mathcal{C})=\phi \mathcal{I}(t)-\left(\omega+\mu_{0}\right) \mathcal{C}(t), \\
& \mathcal{Q}_{3}(t, \mathcal{A})=\rho \mathcal{I}(t)-\left(\alpha+\mu_{0}+d\right) \mathcal{A}(t), \\
& \left\{\begin{array}{l}
\psi_{1}=\mu_{0}+\beta \kappa_{2}+\eta_{c} \kappa_{3}+\eta_{A} \kappa_{4} \\
\psi_{2}=\mu_{0}+\rho+\phi+\beta \kappa_{1} \\
\psi_{3}=\mu_{0}+\omega \\
\psi_{4}=\alpha+d+\mu_{0}
\end{array}\right.
\end{aligned}
$$

- (B) We assume that, for $\mathcal{S}(t), \mathcal{S}^{*}(t), \mathcal{I}(t), \mathcal{I}^{*}(t), \mathcal{C}(t), \mathcal{C}^{*}(t), \mathcal{A}(t), \mathcal{A}^{*}(t) \in L[0,1]$, there exist constants $\kappa_{i}>0$, for $i=1,2,3,4$, such that

$$
\begin{aligned}
\|\mathcal{S}(t)\| \leq \kappa_{1},\|I(t)\| & \leq \kappa_{2},\|C(t)\| \leq \kappa_{3},\|A(t)\| \leq \kappa_{4}, \text { and } \xi_{1}, \xi_{2}>0, \text { and } \\
\|\mathcal{C}(t)+\mathcal{A}(t)\| & \leq \xi_{1}, \\
\|\mathcal{I}(t)+\mathcal{A}(t)\| & \leq \xi_{2} .
\end{aligned}
$$

Theorem 2.1 The $\mathcal{Q}_{i}$ for $i \in \mathcal{N}_{1}^{4}$ satisfies the Lipschitz condition provided that $(\mathcal{B})$ is obeyed.

Consider, for $\mathcal{Q}_{1}$, the following:

$$
\begin{aligned}
\left\|\mathcal{Q}_{1}(t, S)-\mathcal{Q}_{1}\left(t, S^{*}\right)\right\|= & \| \pi-\beta\left(\mathcal{I}(t)-\eta_{c} \mathcal{C}(t)+\eta_{A} \mathcal{A}(t)\right) \mathcal{S}(t)-\mu_{0} \mathcal{S}(t) \\
& -\left(\pi-\beta\left(\mathcal{I}(t)-\eta_{c} \mathcal{C}(t)+\eta_{A} \mathcal{A}(t)\right) \mathcal{S}^{*}(t)-\mu_{0} \mathcal{S}^{*}(t) \|\right. \\
\leq & {\left[\mu_{0}+\beta\|I\|+\eta_{c}\|c\|+\eta_{A}\|A\|\right]\left\|\mathcal{S}-\mathcal{S}^{*}\right\| } \\
\leq & {\left[\mu_{0}+\beta \kappa_{2}+\eta_{c} \kappa_{3}+\eta_{A} \kappa_{4}\right]\left\|\mathcal{S}-\mathcal{S}^{*}\right\| } \\
= & \psi_{1}\left\|\mathcal{S}-\mathcal{S}^{*}\right\| .
\end{aligned}
$$

For $\mathcal{Q}_{2}(t, \mathcal{I})$, we have

$$
\begin{aligned}
&\left\|\mathcal{Q}_{2}(t, \mathcal{I})-\mathcal{Q}_{2}\left(t, \mathcal{I}^{*}\right)\right\| \\
&= \| \beta\left(\mathcal{I}(t)-\eta_{c} \mathcal{C}(t)+\eta_{A} \mathcal{A}(t)\right) \mathcal{S}(t)-\left(\rho+\phi+\mu_{0}\right) \mathcal{I}(t)+\omega \mathcal{C}(t)+\alpha \mathcal{A}(t) \\
&-\left(\beta\left(\mathcal{I}^{*}(t)-\eta_{c} \mathcal{C}(t)+\eta_{A} \mathcal{A}(t)\right) \mathcal{S}(t)-\left(\rho+\phi+\mu_{0}\right) \mathcal{I}^{*}(t)+\omega \mathcal{C}(t)+\alpha \mathcal{A}(t) \|\right. \\
& \leq {\left[\mu_{0}+\rho+\phi+\beta\|\mathcal{S}\|\right]\left\|I-\mathcal{I}^{*}\right\| } \\
& \leq {\left[\mu_{0}+\rho+\phi+\beta \kappa_{1}\right]\left\|I-\mathcal{I}^{*}\right\| } \\
&= \psi_{2}\left\|I-\mathcal{I}^{*}\right\| .
\end{aligned}
$$


$\mathcal{Q}_{3}(t, \mathcal{C})$ implies

$$
\begin{aligned}
\left\|\mathcal{Q}_{3}(t, \mathcal{C})-\mathcal{Q}_{3}\left(t, \mathcal{C}^{*}\right)\right\| & =\| \phi \mathcal{I}(t)-\left(\omega+\mu_{0}\right) \mathcal{C}(t)-\left(\phi \mathcal{I}(t)-\left(\omega+\mu_{0}\right) \mathcal{C}^{*}(t) \|\right. \\
& \leq\left[\mu_{0}+\omega\right]\left\|C-\mathcal{C}^{*}\right\| \\
& =\psi_{3}\left\|C-\mathcal{C}^{*}\right\|
\end{aligned}
$$

And finally, for $\mathcal{Q}_{4}(t, \mathcal{A})$, we have

$$
\begin{aligned}
& \left\|\mathcal{Q}_{4}(t, \mathcal{A})-\mathcal{Q}_{4}\left(t, \mathcal{A}^{*}\right)\right\| \\
& \quad=\left\|\rho \mathcal{I}(t)-\left(\alpha+\mu_{0}+d\right) \mathcal{A}(t)-\left(\rho \mathcal{I}(t)-\left(\alpha+\mu_{0}+d\right) \mathcal{A}^{*}(t)\right)\right\| \\
& \quad \leq\left[\mu_{0}+\alpha+d\right]\left\|A-\mathcal{A}^{*}\right\| \\
& \quad=\psi_{4}\left\|A-\mathcal{A}^{*}\right\| .
\end{aligned}
$$

Thus, from (14) to (17), we have that $\mathcal{Q}_{i}$ for $i=1,2,3,4$ satisfies the Lipschitz condition. This completes the proof.

Assuming that $\mathcal{S}(0)=\mathcal{I}(0)=\mathcal{C}(0)=\mathcal{A}(0)=0$, we have

$$
\begin{aligned}
& \mathcal{S}(t)=\frac{1-\alpha_{1}}{\beta\left(\alpha_{1}\right)} \mathcal{Q}_{1}(t, \mathcal{S}(t))+\frac{\alpha_{1}}{\beta\left(\alpha_{1}\right) \Gamma\left(\alpha_{1}\right)} \int_{0}^{t}(t-s)^{\alpha_{1}-1} \mathcal{Q}_{1}(s, \mathcal{S}(s)) d s \\
& \mathcal{I}(t)=\frac{1-\alpha_{1}}{\beta\left(\alpha_{1}\right)} \mathcal{Q}_{2}(t, \mathcal{I}(t))+\frac{\alpha_{1}}{\beta\left(\alpha_{1}\right) \Gamma\left(\alpha_{1}\right)} \int_{0}^{t}(t-s)^{\alpha_{1}-1} \mathcal{Q}_{2}(s, \mathcal{I}(s)) d s \\
& \mathcal{C}(t)=\frac{1-\alpha_{1}}{\beta\left(\alpha_{1}\right)} \mathcal{Q}_{1}(t, \mathcal{C}(t))+\frac{\alpha_{1}}{\beta\left(\alpha_{1}\right) \Gamma\left(\alpha_{1}\right)} \int_{0}^{t}(t-s)^{\alpha_{1}-1} \mathcal{Q}_{3}(s, \mathcal{C}(s)) d s \\
& \mathcal{A}(t)=\frac{1-\alpha_{1}}{\beta\left(\alpha_{1}\right)} \mathcal{Q}_{1}(t, \mathcal{C}(t))+\frac{\alpha_{1}}{\beta\left(\alpha_{1}\right) \Gamma\left(\alpha_{1}\right)} \int_{0}^{t}(t-s)^{\alpha_{1}-1} \mathcal{Q}_{4}(s, \mathcal{A}(s)) d s
\end{aligned}
$$

For the iterative scheme of model (1), define

$$
\begin{aligned}
& \mathcal{S}_{n}(t)=\frac{1-\alpha_{1}}{\beta\left(\alpha_{1}\right)} \mathcal{Q}_{1}\left(t, \mathcal{S}_{n-1}(t)\right)+\frac{\alpha_{1}}{\beta\left(\alpha_{1}\right) \Gamma\left(\alpha_{1}\right)} \int_{0}^{t}(t-s)^{\alpha_{1}-1} \mathcal{Q}_{1}\left(s, \mathcal{S}_{n-1}(s)\right) d s, \\
& \mathcal{I}_{n}(t)=\frac{1-\alpha_{1}}{\beta\left(\alpha_{1}\right)} \mathcal{Q}_{2}\left(t, \mathcal{I}_{n-1}(t)\right)+\frac{\alpha_{1}}{\beta\left(\alpha_{1}\right) \Gamma\left(\alpha_{1}\right)} \int_{0}^{t}(t-s)^{\alpha_{1}-1} \mathcal{Q}_{2}\left(s, \mathcal{I}_{n-1}(s)\right) d s, \\
& \mathcal{C}_{n}(t)=\frac{1-\alpha_{1}}{\beta\left(\alpha_{1}\right)} \mathcal{Q}_{1}\left(t, \mathcal{C}_{n-1}(t)\right)+\frac{\alpha_{1}}{\beta\left(\alpha_{1}\right) \Gamma\left(\alpha_{1}\right)} \int_{0}^{t}(t-s)^{\alpha_{1}-1} \mathcal{Q}_{3}\left(s, \mathcal{C}_{n-1}(s)\right) d s, \\
& \mathcal{A}_{n}(t)=\frac{1-\alpha_{1}}{\beta\left(\alpha_{1}\right)} \mathcal{Q}_{1}\left(t, \mathcal{C}_{n-1}(t)\right)+\frac{\alpha_{1}}{\beta\left(\alpha_{1}\right) \Gamma\left(\alpha_{1}\right)} \int_{0}^{t}(t-s)^{\alpha_{1}-1} \mathcal{Q}_{4}\left(s, \mathcal{A}_{n-1}(s)\right) d s .
\end{aligned}
$$

Theorem 2.2 The fractional order HIV/AIDS model (1) has a solution if we have

$$
\Delta=\max \left\{\Psi_{i}\right\}<1, \quad i \in \mathcal{N}_{1}^{4} .
$$

We define the function

$$
\mathcal{G} 1_{n}(t)=\mathcal{S}_{n+1}(t)-\mathcal{S}(t), \quad \mathcal{G} 2_{n}(t)=\mathcal{I}_{n+1}(t)-\mathcal{I}(t),
$$




$$
\mathcal{G} 3_{n}(t)=\mathcal{C}_{n+1}(t)-\mathcal{C}(t), \quad \mathcal{G} 4_{n}(t)=\mathcal{A}_{n+1}(t)-\mathcal{A}(t) .
$$

Then, using equations (3) to (24), we find that

$$
\begin{aligned}
\left\|\mathcal{G} 1_{n}\right\| \leq & \frac{1-\alpha_{1}}{\beta\left(\alpha_{1}\right)}\left\|\mathcal{Q}_{1}\left(t, \mathcal{S}_{n}(t)\right)-\mathcal{Q}_{1}\left(t, \mathcal{S}_{n-1}(t)\right)\right\| \\
& +\frac{\alpha_{1}}{\beta\left(\alpha_{1}\right) \Gamma\left(\alpha_{1}\right)} \int_{0}^{t}(t-s)^{\alpha_{1}-1}\left\|\mathcal{Q}_{1}\left(s, \mathcal{S}_{n}(s)\right)-\mathcal{Q}_{1}\left(t, \mathcal{S}_{n-1}(t)\right)\right\| d s \\
\leq & {\left[\frac{1-\alpha_{1}}{\beta\left(\alpha_{1}\right)}+\frac{1}{\beta\left(\alpha_{1}\right) \Gamma\left(\alpha_{1}\right)}\right] \psi_{1}\left\|\mathcal{S}_{n}-S\right\| } \\
\leq & {\left[\frac{1-\alpha_{1}}{\beta\left(\alpha_{1}\right)}+\frac{1}{\beta\left(\alpha_{1}\right) \Gamma\left(\alpha_{1}\right)}\right]^{n} \Delta^{n}\left\|\mathcal{S}_{n}-\mathcal{S}\right\| . }
\end{aligned}
$$

And

$$
\begin{aligned}
\left\|\mathcal{G} 2_{n}\right\| \leq & \frac{1-\alpha_{1}}{\beta\left(\alpha_{1}\right)}\left\|\mathcal{Q}_{2}\left(t, \mathcal{I}_{n}(t)\right)-\mathcal{Q}_{2}\left(t, \mathcal{I}_{n-1}(t)\right)\right\| \\
& +\frac{\alpha_{1}}{\beta\left(\alpha_{1}\right) \Gamma\left(\alpha_{1}\right)} \int_{0}^{t}(t-s)^{\alpha_{1}-1}\left\|\mathcal{Q}_{2}\left(s, \mathcal{I}_{n}(s)\right)-\mathcal{Q}_{2}\left(t, \mathcal{I}_{n-1}(t)\right)\right\| d s \\
\leq & {\left[\frac{1-\alpha_{1}}{\beta\left(\alpha_{1}\right)}+\frac{1}{\beta\left(\alpha_{1}\right) \Gamma\left(\alpha_{1}\right)}\right] \psi_{2}\left\|\mathcal{I}_{n}-I\right\| } \\
\leq & {\left[\frac{1-\alpha_{1}}{\beta\left(\alpha_{1}\right)}+\frac{1}{\beta\left(\alpha_{1}\right) \Gamma\left(\alpha_{1}\right)}\right]^{n} \Delta^{n}\left\|\mathcal{I}_{n}-I\right\| . }
\end{aligned}
$$

Similarly,

$$
\begin{aligned}
\left\|\mathcal{G}_{n}\right\| \leq & \frac{1-\alpha_{1}}{\beta\left(\alpha_{1}\right)}\left\|\mathcal{Q}_{3}\left(t, \mathcal{C}_{n}(t)\right)-\mathcal{Q}_{3}\left(t, \mathcal{C}_{n-1}(t)\right)\right\| \\
& +\frac{\alpha_{1}}{\beta\left(\alpha_{1}\right) \Gamma\left(\alpha_{1}\right)} \int_{0}^{t}(t-s)^{\alpha_{1}-1}\left\|\mathcal{Q}_{3}\left(s, \mathcal{C}_{n}(s)\right)-\mathcal{Q}_{3}\left(t, \mathcal{C}_{n-1}(t)\right)\right\| d s \\
\leq & {\left[\frac{1-\alpha_{1}}{\beta\left(\alpha_{1}\right)}+\frac{1}{\beta\left(\alpha_{1}\right) \Gamma\left(\alpha_{1}\right)}\right] \psi_{3}\left\|\mathcal{C}_{n}-\mathcal{C}\right\| } \\
\leq & {\left[\frac{1-\alpha_{1}}{\beta\left(\alpha_{1}\right)}+\frac{1}{\beta\left(\alpha_{1}\right) \Gamma\left(\alpha_{1}\right)}\right]^{n} \Delta^{n}\left\|\mathcal{C}_{n}-\mathcal{C}\right\|, } \\
\left\|\mathcal{G} 4_{n}\right\| \leq & \frac{1-\alpha_{1}}{\beta\left(\alpha_{1}\right)}\left\|\mathcal{Q}_{4}\left(t, \mathcal{A}_{n}(t)\right)-\mathcal{Q}_{4}\left(t, \mathcal{A}_{n-1}(t)\right)\right\| \\
& +\frac{\alpha_{1}}{\beta\left(\alpha_{1}\right) \Gamma\left(\alpha_{1}\right)} \int_{0}^{t}(t-s)^{\alpha_{1}-1}\left\|\mathcal{Q}_{4}\left(s, \mathcal{\mathcal { A } _ { n }}(s)\right)-\mathcal{Q}_{4}\left(t, \mathcal{A}_{n-1}(t)\right)\right\| d s \\
\leq & {\left[\frac{1-\alpha_{1}}{\beta\left(\alpha_{1}\right)}+\frac{1}{\beta\left(\alpha_{1}\right) \Gamma\left(\alpha_{1}\right)}\right]^{\psi_{4}\left\|\mathcal{A}_{n}-\mathcal{A}\right\|} } \\
\leq & {\left[\frac{1-\alpha_{1}}{\beta\left(\alpha_{1}\right)}+\frac{1}{\beta\left(\alpha_{1}\right) \Gamma\left(\alpha_{1}\right)}\right]^{n} \Delta^{n}\left\|\mathcal{A}_{n}-\mathcal{A}\right\| . }
\end{aligned}
$$

Thus, we have $\mathcal{G}(t)_{n} \rightarrow 0, i \in \mathcal{N}_{1}^{4}$, as $n \rightarrow \infty$ for $\Delta<1$, which completes the proof.

For our suggested model (1), we study the uniqueness of solution. 
Theorem 3.1 HIV/AIDS model (1) has a unique solution if

$$
\left[\frac{1-\alpha_{i}}{\beta\left(\alpha_{i}\right)}+\frac{1}{\beta\left(\alpha_{i}\right) \Gamma\left(\alpha_{i}\right)}\right] \psi_{i} \leq 1, \quad i \in \mathcal{N}_{1}^{4}
$$

Let there exist another solution $\overline{\mathcal{S}}(t), \overline{\mathcal{I}}(t), \overline{\mathcal{C}}(t), \overline{\mathcal{A}}(t)$ such that

$$
\begin{aligned}
& \overline{\mathcal{S}}(t)=\frac{1-\alpha_{1}}{\beta\left(\alpha_{1}\right)} \mathcal{Q}_{1}(t, \overline{\mathcal{S}}(t))+\frac{\alpha_{1}}{\beta\left(\alpha_{1}\right) \Gamma\left(\alpha_{1}\right)} \int_{0}^{t}(t-s)^{\alpha_{1}-1} \mathcal{Q}_{1}(s, \overline{\mathcal{S}}(s)) d s \\
& \overline{\mathcal{I}}(t)=\frac{1-\alpha_{1}}{\beta\left(\alpha_{1}\right)} \mathcal{Q}_{2}(t, \overline{\mathcal{I}}(t))+\frac{\alpha_{1}}{\beta\left(\alpha_{1}\right) \Gamma\left(\alpha_{1}\right)} \int_{0}^{t}(t-s)^{\alpha_{1}-1} \mathcal{Q}_{2}(s, \overline{\mathcal{I}}(s)) d s \\
& \overline{\mathcal{C}}(t)=\frac{1-\alpha_{1}}{\beta\left(\alpha_{1}\right)} \mathcal{Q}_{3}(t, \overline{\mathcal{C}}(t))+\frac{\alpha_{1}}{\beta\left(\alpha_{1}\right) \Gamma\left(\alpha_{1}\right)} \int_{0}^{t}(t-s)^{\alpha_{1}-1} \mathcal{Q}_{3}(s, \overline{\mathcal{C}}(s)) d s \\
& \overline{\mathcal{A}}(t)=\frac{1-\alpha_{1}}{\beta\left(\alpha_{1}\right)} \mathcal{Q}_{4}(t, \overline{\mathcal{C}}(t))+\frac{\alpha_{1}}{\beta\left(\alpha_{1}\right) \Gamma\left(\alpha_{1}\right)} \int_{0}^{t}(t-s)^{\alpha_{1}-1} \mathcal{Q}_{4}(s, \overline{\mathcal{A}}(s)) d s
\end{aligned}
$$

Then

$$
\begin{aligned}
\|\mathcal{S}(t)-\overline{\mathcal{S}}(t)\| \leq & \frac{1-\alpha_{1}}{\beta\left(\alpha_{1}\right)}\left\|\mathcal{Q}_{1}(t, \mathcal{S}(t))-\mathcal{Q}_{1}(t, \overline{\mathcal{S}}(t))\right\| \\
& +\frac{\alpha_{1}}{\beta\left(\alpha_{1}\right) \Gamma\left(\alpha_{1}\right)} \int_{0}^{t}(t-s)^{\alpha_{1}-1}\left\|\mathcal{Q}_{1}(s, \mathcal{S}(s))-\mathcal{Q}_{1}(t, \overline{\mathcal{S}}(t))\right\| d s \\
\leq & {\left[\frac{1-\alpha_{1}}{\beta\left(\alpha_{1}\right)}+\frac{1}{\beta\left(\alpha_{1}\right) \Gamma\left(\alpha_{1}\right)}\right] \psi_{1}\|\mathcal{S}-\overline{\mathcal{S}}\|, }
\end{aligned}
$$

which implies

$$
\left[\frac{1-\alpha_{1}}{\beta\left(\alpha_{1}\right)} \psi_{1}+\frac{\psi_{1}}{\beta\left(\alpha_{1}\right) \Gamma\left(\alpha_{1}\right)}-1\right]\left\|\mathcal{S}_{-} \overline{\mathcal{S}}\right\| \geq 0
$$

By (29), (35) is true if $\|\mathcal{S}-\overline{\mathcal{S}}\|=0$, which implies $\mathcal{S}(t)=\overline{\mathcal{S}}(t)$. Similarly, we have

$$
\begin{aligned}
\|\mathcal{I}(t)-\overline{\mathcal{I}}(t)\| \leq & \frac{1-\alpha_{1}}{\beta\left(\alpha_{1}\right)}\left\|\mathcal{Q}_{2}(t, \mathcal{I}(t))-\mathcal{Q}_{2}(t, \overline{\mathcal{I}}(t))\right\| \\
& +\frac{\alpha_{1}}{\beta\left(\alpha_{1}\right) \Gamma\left(\alpha_{1}\right)} \int_{0}^{t}(t-s)^{\alpha_{1}-1}\left\|\mathcal{Q}_{2}(s, \mathcal{C}(s))-\mathcal{Q}_{2}(t, \overline{\mathcal{I}}(t))\right\| d s \\
\leq & {\left[\frac{1-\alpha_{1}}{\beta\left(\alpha_{1}\right)}+\frac{1}{\beta\left(\alpha_{1}\right) \Gamma\left(\alpha_{1}\right)}\right] \psi_{2}\|\mathcal{C}-\overline{\mathcal{C}}\|, }
\end{aligned}
$$

which implies

$$
\left[\frac{1-\alpha_{1}}{\beta\left(\alpha_{1}\right)} \psi_{1}+\frac{\psi_{1}}{\beta\left(\alpha_{1}\right) \Gamma\left(\alpha_{1}\right)}-1\right]\left\|\mathcal{I}_{-} \overline{\mathcal{I}}\right\| \geq 0
$$

By (29), (37) is true if $\|\mathcal{I}-\overline{\mathcal{I}}\|=0$, which implies $\mathcal{I}(t)=\overline{\mathcal{I}}(t)$. Now, for $\mathcal{C}$, we have

$$
\|\mathcal{C}(t)-\overline{\mathcal{C}}(t)\| \leq \frac{1-\alpha_{1}}{\beta\left(\alpha_{1}\right)}\left\|\mathcal{Q}_{3}(t, \mathcal{C}(t))-\mathcal{Q}_{3}(t, \overline{\mathcal{C}}(t))\right\|
$$




$$
\begin{aligned}
& +\frac{\alpha_{1}}{\beta\left(\alpha_{1}\right) \Gamma\left(\alpha_{1}\right)} \int_{0}^{t}(t-s)^{\alpha_{1}-1}\left\|\mathcal{Q}_{3}(s, \mathcal{C}(s))-\mathcal{Q}_{3}(t, \overline{\mathcal{C}}(t))\right\| d s \\
\leq & {\left[\frac{1-\alpha_{1}}{\beta\left(\alpha_{1}\right)}+\frac{1}{\beta\left(\alpha_{1}\right) \Gamma\left(\alpha_{1}\right)}\right] \psi_{2}\|\mathcal{C}-\overline{\mathcal{C}}\|, }
\end{aligned}
$$

which implies

$$
\left[\frac{1-\alpha_{1}}{\beta\left(\alpha_{1}\right)} \psi_{1}+\frac{\psi_{1}}{\beta\left(\alpha_{1}\right) \Gamma\left(\alpha_{1}\right)}-1\right]\left\|\mathcal{C}_{-} \overline{\mathcal{C}}\right\| \geq 0
$$

By (29), (39) is true if $\|\mathcal{C}-\overline{\mathcal{C}}\|=0$, which implies $\mathcal{C}(t)=\overline{\mathcal{C}}(t)$. Similarly, $\mathcal{A}(t)=\overline{\mathcal{A}}(t)$. Thus (1) has a unique solution.

\section{Hyers-Ulam stability}

Definition 4.1 The integral system (18)-(21) is Hyers-Ulam stable if, for $\Delta_{i}>0, i \in \mathcal{N}_{1}^{4}$ and $\gamma_{i}>0, i \in \mathcal{N}_{1}^{4}$ such that

$$
\begin{aligned}
& \left|\mathcal{S}(t)-\frac{1-\alpha_{1}}{\beta\left(\alpha_{1}\right)} \mathcal{Q}_{1}(t, \mathcal{S}(t))-\frac{\alpha_{1}}{\beta\left(\alpha_{1}\right) \Gamma\left(\alpha_{1}\right)} \int_{0}^{t}(t-s)^{\alpha_{1}-1} \mathcal{Q}_{1}(s, \mathcal{S}(s)) d s\right| \leq \gamma_{1}, \\
& \left|\mathcal{I}(t)-\frac{1-\alpha_{1}}{\beta\left(\alpha_{1}\right)} \mathcal{Q}_{2}(t, \mathcal{I}(t))-\frac{\alpha_{1}}{\beta\left(\alpha_{1}\right) \Gamma\left(\alpha_{1}\right)} \int_{0}^{t}(t-s)^{\alpha_{1}-1} \mathcal{Q}_{2}(s, \mathcal{I}(s)) d s\right| \leq \gamma_{2}, \\
& \left|\mathcal{C}(t)-\frac{1-\alpha_{1}}{\beta\left(\alpha_{1}\right)} \mathcal{Q}_{3}(t, \mathcal{C}(t))-\frac{\alpha_{1}}{\beta\left(\alpha_{1}\right) \Gamma\left(\alpha_{1}\right)} \int_{0}^{t}(t-s)^{\alpha_{1}-1} \mathcal{Q}_{3}(s, \mathcal{C}(s)) d s\right| \leq \gamma_{3}, \\
& \left|\mathcal{A}(t)-\frac{1-\alpha_{1}}{\beta\left(\alpha_{1}\right)} \mathcal{Q}_{4}(t, \mathcal{A}(t))-\frac{\alpha_{1}}{\beta\left(\alpha_{1}\right) \Gamma\left(\alpha_{1}\right)} \int_{0}^{t}(t-s)^{\alpha_{1}-1} \mathcal{Q}_{4}(s, \mathcal{A}(s)) d s\right| \leq \gamma_{4},
\end{aligned}
$$

we have $\dot{\mathcal{S}}(t), \dot{\mathcal{I}}(t), \dot{\mathcal{C}}(t), \dot{\mathcal{A}}(t)$, which implies

$$
\begin{aligned}
& \dot{\mathcal{S}}(t)=\frac{1-\alpha_{1}}{\beta\left(\alpha_{1}\right)} \mathcal{Q}_{1}(t, \dot{\mathcal{S}}(t))+\frac{\alpha_{1}}{\beta\left(\alpha_{1}\right) \Gamma\left(\alpha_{1}\right)} \int_{0}^{t}(t-s)^{\alpha_{1}-1} \mathcal{Q}_{1}(s, \dot{\mathcal{S}}(s)) d s, \\
& \dot{\mathcal{I}}(t)=\frac{1-\alpha_{1}}{\beta\left(\alpha_{1}\right)} \mathcal{Q}_{2}(t, \dot{\mathcal{I}}(t))+\frac{\alpha_{1}}{\beta\left(\alpha_{1}\right) \Gamma\left(\alpha_{1}\right)} \int_{0}^{t}(t-s)^{\alpha_{1}-1} \mathcal{Q}_{2}(s, \dot{\mathcal{I}}(s)) d s, \\
& \dot{\mathcal{C}}(t)=\frac{1-\alpha_{1}}{\beta\left(\alpha_{1}\right)} \mathcal{Q}_{3}(t, \dot{\mathcal{C}}(t))+\frac{\alpha_{1}}{\beta\left(\alpha_{1}\right) \Gamma\left(\alpha_{1}\right)} \int_{0}^{t}(t-s)^{\alpha_{1}-1} \mathcal{Q}_{3}(s, \dot{\mathcal{C}}(s)) d s, \\
& \dot{\mathcal{A}}(t)=\frac{1-\alpha_{1}}{\beta\left(\alpha_{1}\right)} \mathcal{Q}_{4}(t, \dot{\mathcal{A}}(t))+\frac{\alpha_{1}}{\beta\left(\alpha_{1}\right) \Gamma\left(\alpha_{1}\right)} \int_{0}^{t}(t-s)^{\alpha_{1}-1} \mathcal{Q}_{4}(s, \dot{\mathcal{A}}(s)) d s
\end{aligned}
$$

such that

$$
\begin{aligned}
|\mathcal{S}(t)-\dot{\mathcal{S}}(t)| \leq \delta_{1} \gamma_{1}, & |\mathcal{I}(t)-\dot{\mathcal{I}}(t)| \leq \delta_{2} \gamma_{2}, \\
|\mathcal{C}(t)-\dot{\mathcal{C}}(t)| \leq \delta_{3} \gamma_{3}, & |\mathcal{A}(t)-\dot{\mathcal{A}}(t)| \leq \delta_{4} \gamma_{4} .
\end{aligned}
$$

Theorem 4.2 Let $(\mathcal{B})$ be satisfied. Then (1) is Hyers-Ulam stable.

Proof By Theorem 3.1, HIV/AIDS model (1) has a unique solution, say $\mathcal{S}(t), \mathcal{I}(t), \mathcal{C}(t), \mathcal{A}(t)$. Let $(\dot{\mathcal{S}}(t), \dot{\mathcal{I}}(t), \dot{\mathcal{C}}(t), \dot{\mathcal{A}}(t)$ be an approximate solution of (1) satisfying (18)-(21). Then we 
have

$$
\begin{aligned}
\|\mathcal{S}(t)-\dot{\mathcal{S}}(t)\| \leq & \frac{1-\alpha_{1}}{\beta\left(\alpha_{1}\right)}\left\|\mathcal{Q}_{1}(t, \mathcal{S}(t))-\mathcal{Q}_{1}(t, \dot{\mathcal{S}}(t))\right\| \\
& +\frac{\alpha_{1}}{\beta\left(\alpha_{1}\right) \Gamma\left(\alpha_{1}\right)} \int_{0}^{t}(t-s)^{\alpha_{1}-1}\left\|\mathcal{Q}_{1}(s, \mathcal{S}(s))-\mathcal{Q}_{1}(t, \dot{\mathcal{S}}(t))\right\| d s \\
\leq & {\left[\frac{1-\alpha_{1}}{\beta\left(\alpha_{1}\right)}+\frac{1}{\beta\left(\alpha_{1}\right) \Gamma\left(\alpha_{1}\right)}\right] \psi_{1}\|\mathcal{S}-\dot{\mathcal{S}}\| . }
\end{aligned}
$$

Taking $\gamma_{1}=\psi_{1}, \Delta=\frac{1-\alpha_{1}}{\beta\left(\alpha_{1}\right)}+\frac{\alpha_{1}}{\beta\left(\alpha_{1}\right) \Gamma\left(\alpha_{1}\right)}$, this implies

$$
\|\mathcal{S}(t)-\dot{\mathcal{S}}(t)\| \leq \gamma_{1} \Delta_{1} .
$$

Similarly, for $\mathcal{I}(t), \dot{\mathcal{I}}, \mathcal{C}(t), \dot{\mathcal{C}}(t), \mathcal{A}(t), \dot{\mathcal{A}}(t)$, we have

$$
\left\{\begin{array}{l}
\|\mathcal{I}(t)-\dot{\mathcal{I}}(t)\| \leq \gamma_{2} \Delta, \\
\|\mathcal{C}(t)-\dot{\mathcal{C}}(t)\| \leq \gamma_{3} \Delta, \\
\|\mathcal{A}(t)-\dot{\mathcal{A}}(t)\| \leq \gamma_{4} \Delta .
\end{array}\right.
$$

This implies that system (1) is Hyers-Ulam stable, which ultimately ensures the stability of (1). This completes the proof.

\section{Numerical scheme}

With the help of (7)-(10), we produce the following numerical scheme:

$$
\left\{\begin{array}{l}
{ }_{0}^{A B C} \mathcal{D}_{t}^{\alpha_{1}} \mathcal{S}(t)=\mathcal{Q}_{1}(t, \mathcal{S}), \\
{ }_{0}^{A B C} \mathcal{D}_{t}^{\alpha_{1}} \mathcal{I}(t)=\mathcal{Q}_{2}(t . \mathcal{I}), \\
{ }_{0}^{A B C} \mathcal{D}_{t}^{\alpha_{1}} \mathcal{C}(t)=\mathcal{Q}_{3}(t, \mathcal{C}), \\
{ }_{0}^{A B C} \mathcal{D}_{t}^{\alpha_{1}} \mathcal{A}(t)=\mathcal{Q}_{4}(t, \mathcal{A}) .
\end{array}\right.
$$

With the help of fractional AB-integral operator, (51) gets the following form:

$$
\begin{aligned}
& \mathcal{S}(t)-\mathcal{S}(0)=\frac{1-\alpha_{1}}{\beta\left(\alpha_{1}\right)} \mathcal{Q}_{1}(t, \mathcal{S})+\frac{\alpha_{1}}{\beta\left(\alpha_{1}\right) \Gamma\left(\alpha_{1}\right)} \int_{0}^{t}(t-s)^{\alpha_{1}-1} \mathcal{Q}_{1}(s, \mathcal{S}) d s \\
& \mathcal{I}(t)-\mathcal{I}(0)=\frac{1-\alpha_{1}}{\beta\left(\alpha_{1}\right)} \mathcal{Q}_{2}(t, \mathcal{I})+\frac{\alpha_{1}}{\beta\left(\alpha_{1}\right) \Gamma\left(\alpha_{1}\right)} \int_{0}^{t}(t-s)^{\alpha_{1}-1} \mathcal{Q}_{2}(s, \mathcal{I}) d s \\
& \mathcal{C}(t)-\mathcal{C}(0)=\frac{1-\alpha_{1}}{\beta\left(\alpha_{1}\right)} \mathcal{Q}_{3}(t, \mathcal{C})+\frac{\alpha_{1}}{\beta\left(\alpha_{1}\right) \Gamma\left(\alpha_{1}\right)} \int_{0}^{t}(t-s)^{\alpha_{1}-1} \mathcal{Q}_{3}(s, \mathcal{C}) d s \\
& \mathcal{A}(t)-\mathcal{A}(0)=\frac{1-\alpha_{1}}{\beta\left(\alpha_{1}\right)} \mathcal{Q}_{4}(t, \mathcal{A})+\frac{\alpha_{1}}{\beta\left(\alpha_{1}\right) \Gamma\left(\alpha_{1}\right)} \int_{0}^{t}(t-s)^{\alpha_{1}-1} \mathcal{Q}_{4}(s, \mathcal{A}) d s
\end{aligned}
$$

By dividing the assumed interval $[0, t]$ into subintervals with the help of point $t_{n+1}$, for $n=0,1,2 \ldots$, we have

$$
\mathcal{S}\left(t_{n+1}\right)-\mathcal{S}(0)
$$




$$
\begin{aligned}
& \quad=\frac{1-\alpha_{1}}{\beta\left(\alpha_{1}\right)} \mathcal{Q}_{1}\left(t_{n}, \mathcal{S}\right)+\frac{\alpha_{1}}{\beta\left(\alpha_{1}\right) \Gamma\left(\alpha_{1}\right)} \sum_{k=0}^{n} \int_{t_{k}}^{t_{k+1}}\left(t_{n+1}-s\right)^{\alpha_{1}-1} \mathcal{Q}_{1}(s, \mathcal{S}) d s \\
& \mathcal{I}\left(t_{n+1}\right)-\mathcal{I}(0) \\
& \quad=\frac{1-\alpha_{1}}{\beta\left(\alpha_{1}\right)} \mathcal{Q}_{2}\left(t_{n}, \mathcal{I}\right)+\frac{\alpha_{1}}{\beta\left(\alpha_{1}\right) \Gamma\left(\alpha_{1}\right)} \sum_{k=0}^{n} \int_{t_{k}}^{t_{k+1}}\left(t_{n+1}-s\right)^{\alpha_{1}-1} \mathcal{Q}_{2}(s, \mathcal{I}) d s \\
& \mathcal{C}\left(t_{n+1}\right)-\mathcal{C}(0) \\
& \quad=\frac{1-\alpha_{1}}{\beta\left(\alpha_{1}\right)} \mathcal{Q}_{3}\left(t_{n}, \mathcal{C}\right)+\frac{\alpha_{1}}{\beta\left(\alpha_{1}\right) \Gamma\left(\alpha_{1}\right)} \sum_{k=0}^{n} \int_{t_{k}}^{t_{k+1}}\left(t_{n+1}-s\right)^{\alpha_{1}-1} \mathcal{Q}_{3}(s, \mathcal{C}) d s \\
& \mathcal{A}\left(t_{n+1}\right)-\mathcal{A}(0) \\
& \quad=\frac{1-\alpha_{1}}{\beta\left(\alpha_{1}\right)} \mathcal{Q}_{4}\left(t_{n}, \mathcal{A}\right)+\frac{\alpha_{1}}{\beta\left(\alpha_{1}\right) \Gamma\left(\alpha_{1}\right)} \sum_{k=0}^{n} \int_{t_{k}}^{t_{k+1}}\left(t_{n+1}-s\right)^{\alpha_{1}-1} \mathcal{Q}_{4}(s, \mathcal{A}) d s
\end{aligned}
$$

Now, using the Lagrange interpolation, we have

$$
\begin{aligned}
& \mathcal{S}\left(t_{n+1}\right)=\mathcal{S}(0)+\frac{1-\alpha_{1}}{\beta\left(\alpha_{1}\right)} \mathcal{Q}_{1}\left(t_{k}, \mathcal{S}\right)+\frac{\alpha_{1}}{\mathcal{B}\left(\alpha_{1}\right)} \\
& \times \sum_{k=0}^{n}\left[\frac { h ^ { \alpha _ { 1 } } \mathcal { Q } _ { 1 } ( t _ { k } , \mathcal { S } ) } { \Gamma ( \alpha _ { 1 } + 2 ) } \left((n+1-k)^{\alpha_{1}}\left(n-k+2+\alpha_{1}\right)\right.\right. \\
& \left.-(n-k)^{\alpha_{1}}\left(n-k+2+2 \alpha_{1}\right)\right)-\frac{h^{\alpha_{1}} \mathcal{Q}_{1}\left(t_{k-1}, \mathcal{S}\right)}{\Gamma\left(\alpha_{1}+2\right)} \\
& \left.\times\left((n+1-k)^{\alpha_{1}}-(n-k)^{\alpha_{1}}\left(n+1-k+\alpha_{1}\right)\right)\right], \\
& \mathcal{I}\left(t_{n+1}\right)=\mathcal{I}(0)+\frac{1-\alpha_{1}}{\beta\left(\alpha_{1}\right)} \mathcal{Q}_{2}\left(t_{k}, \mathcal{I}\right)+\frac{\alpha_{1}}{\mathcal{B}\left(\alpha_{1}\right)} \\
& \times \sum_{k=0}^{n}\left[\frac { h ^ { \alpha _ { 1 } } \mathcal { Q } _ { 2 } ( t _ { k } , \mathcal { I } ) } { \Gamma ( \alpha _ { 1 } + 2 ) } \left((n+1-k)^{\alpha_{1}}\left(n-k+2+\alpha_{1}\right)\right.\right. \\
& \left.-(n-k)^{\alpha_{1}}\left(n-k+2+2 \alpha_{1}\right)\right)-\frac{h^{\alpha_{1}} \mathcal{Q}_{2}\left(t_{k-1}, \mathcal{I}\right)}{\Gamma\left(\alpha_{1}+2\right)} \\
& \left.\times\left((n-k+1)^{\alpha_{1}}-(n-k)^{\alpha_{1}}\left(n+1-k+\alpha_{1}\right)\right)\right], \\
& \mathcal{C}\left(t_{n+1}\right)=\mathcal{C}(0)+\frac{1-\alpha_{1}}{\beta\left(\alpha_{1}\right)} \mathcal{Q}_{3}\left(t_{k}, \mathcal{C}\right)+\frac{\alpha_{1}}{\mathcal{B}\left(\alpha_{1}\right)} \\
& \times \sum_{k=0}^{n}\left[\frac { h ^ { \alpha _ { 1 } } \mathcal { Q } _ { 3 } ( t _ { k } , \mathcal { I } ) } { \Gamma ( \alpha _ { 1 } + 2 ) } \left((n+1-k)^{\alpha_{1}}\left(n-k+2+\alpha_{1}\right)\right.\right. \\
& \left.-(n-k)^{\alpha_{1}}\left(n-k+2+2 \alpha_{1}\right)\right)-\frac{h^{\alpha_{1}} \mathcal{Q}_{3}\left(t_{k-1}, \mathcal{C}\right)}{\Gamma\left(\alpha_{1}+2\right)} \\
& \left.\times\left((n+1-k)^{\alpha_{1}}-(n-k)^{\alpha_{1}}\left(n+1-k+\alpha_{1}\right)\right)\right], \\
& \mathcal{A}\left(t_{n+1}\right)=\mathcal{A}(0)+\frac{1-\alpha_{1}}{\beta\left(\alpha_{1}\right)} \mathcal{Q}_{4}\left(t_{k}, \mathcal{A}\right)+\frac{\alpha_{1}}{\mathcal{B}\left(\alpha_{1}\right)}
\end{aligned}
$$




$$
\begin{aligned}
& \times \sum_{k=0}^{n}\left[\frac { h ^ { \alpha _ { 1 } } \mathcal { Q } _ { 4 } ( t _ { k } , \mathcal { A } ) } { \Gamma ( \alpha _ { 1 } + 2 ) } \left((n+1-k)^{\alpha_{1}}\left(n-k+2+\alpha_{1}\right)\right.\right. \\
& \left.-(n-k)^{\alpha_{1}}\left(n-k+2+2 \alpha_{1}\right)\right)-\frac{h^{\alpha_{1}} \mathcal{Q}_{4}\left(t_{k-1}, \mathcal{A}\right)}{\Gamma\left(\alpha_{1}+2\right)} \\
& \left.\times\left((n+1-k)^{\alpha_{1}+1}-(n-k)^{\alpha_{1}}\left(n+1-k+\alpha_{1}\right)\right)\right] .
\end{aligned}
$$

\section{Computational results}

In this subsection, we present the numerical results with the help of several plots. These results have been produced as per the numerical scheme discussed above. In the computation, we have considered the parametric values as follows: $\mu_{0}=\frac{1}{70}, \beta=0.001, \Lambda=2$, $\eta_{A}=1.3, \eta_{C}=0.04, \omega=0.09, \phi=0.1, \rho=0.1, \alpha=0.33, d=1$.

The objective of the present study of an HIV disease is to describe the transmission process of the disease that can be biologically interpreted as follows: when infectious people of HIV enter a population of potential people, the disease is transmitted to other individuals through the mode of transmission of HIV. An individual who is suffering from HIV may remain asymptomatic at the early stage of infection, only later showing the onset of clinical symptoms and being diagnosed as a disease case.

Illustrative graphs show that the epidemiology of HIV is broadly predictable. Figure 1, represents the comparison of the susceptable class for orders $1,0.98,0.96,0.94$, while keeping the $h=0.09$. Figure 2, represents the comparison of the susceptable class for orders $1,0.98,0.96,0.94$, while keeping the $h=0.09$. This class shows an increase in the number of people with respect to the time due to the decrease in infection $\mathcal{I}(t)$ as given in the Fig. 3 and recury given in Fig. 2. Our model reflects that if the treatment is continued the number of AIDS can be reduced with respect to the time as given in Fig. 4. Figures 5-8, are the joint solutions of the model.

We have determined conditions for when the disease persists and when it can be eradicated. Graphs of various populations with different derivative orders are reflected in each variable of our model, which serves as a proxy for variations in the susceptible, suscepti-

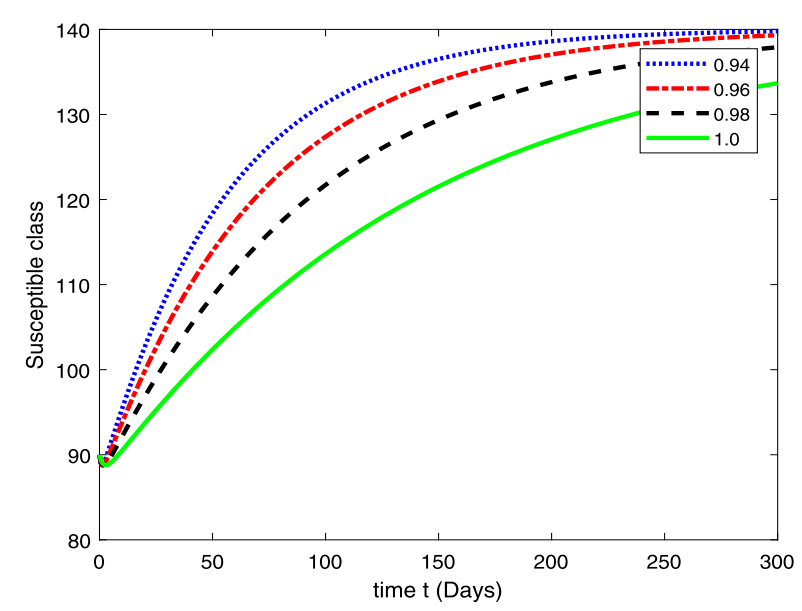

Figure 1 The susceptible class $\mathcal{S}(t)$ for $\alpha_{1}=1,0.98,0.96,0.94$ and $h=0.09$ 


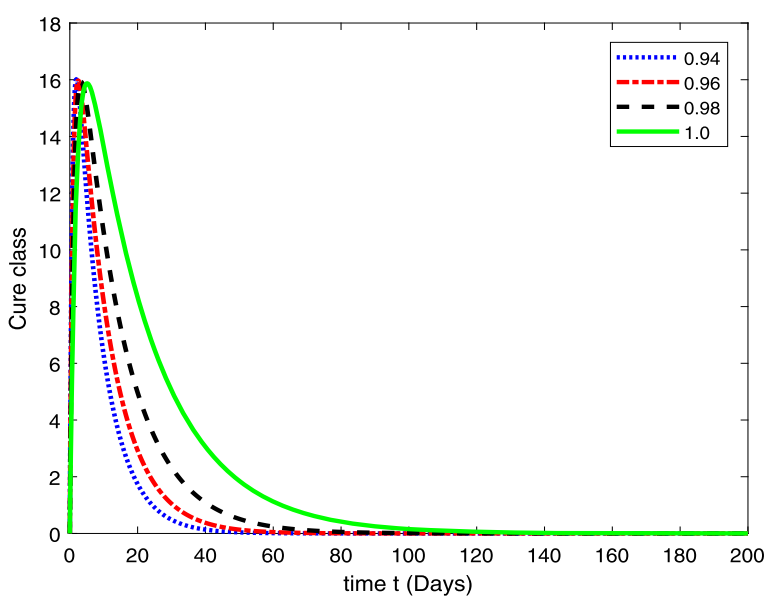

Figure 2 The cured class $\mathcal{C}(t)$ for $\alpha_{1}=1,0.98,0.96,0.94$ and $h=0.09$

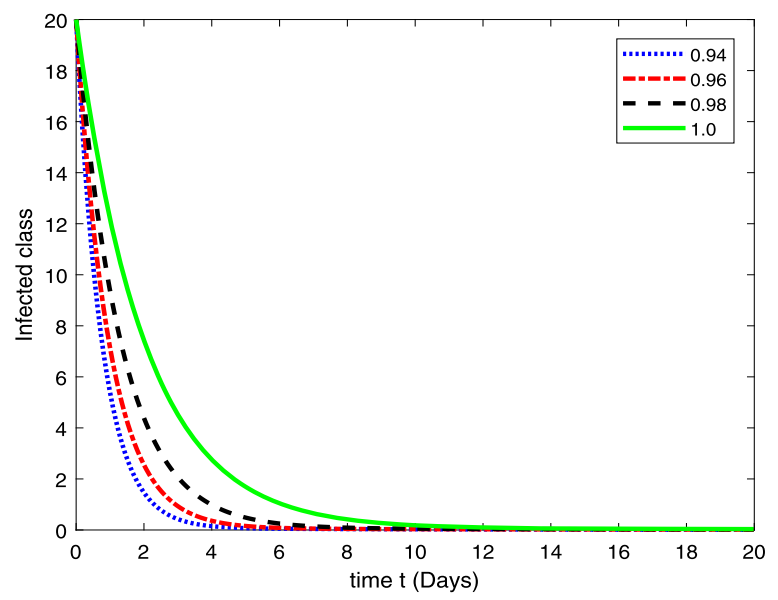

Figure 3 The infected class $\mathcal{I}(t)$ for $\alpha_{1}=1,0.98,0.96,0.94$ and $h=0.09$

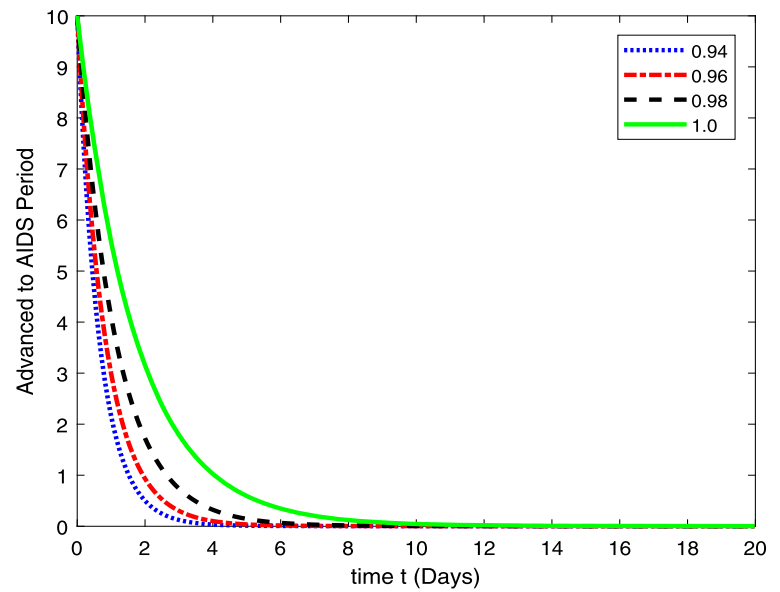

Figure 4 The AIDS class $\mathcal{A}(t)$ for $\alpha_{1}=1,0.98,0.96,0.94$ and $h=0.09$ 


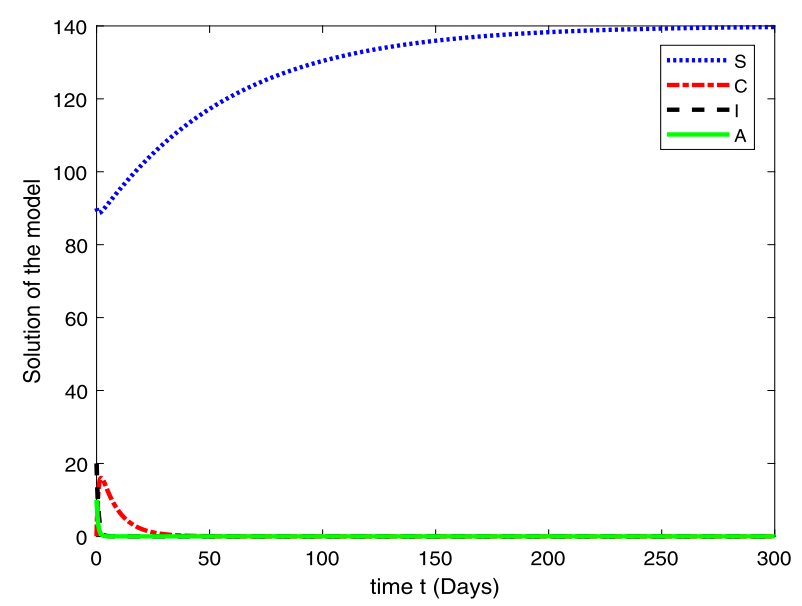

Figure 5 The solution for (1) for $\mathcal{S}, \mathcal{I}, \mathcal{C}, \mathcal{A}$, keeping $h=0.1$ and $\alpha=1$

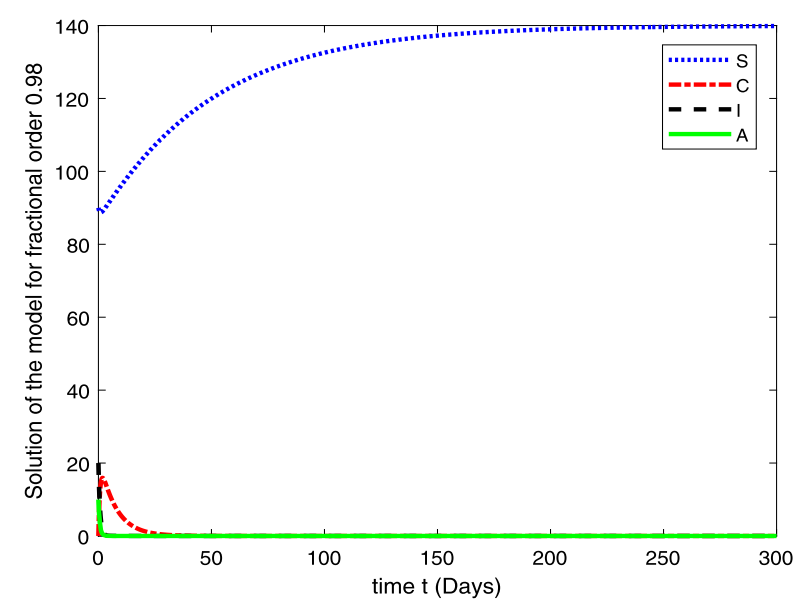

Figure 6 The solution for (1) for $\mathcal{S}, \mathcal{I}, \mathcal{C}, \mathcal{A}$, keeping $h=0.1$ and $\alpha=0.98$

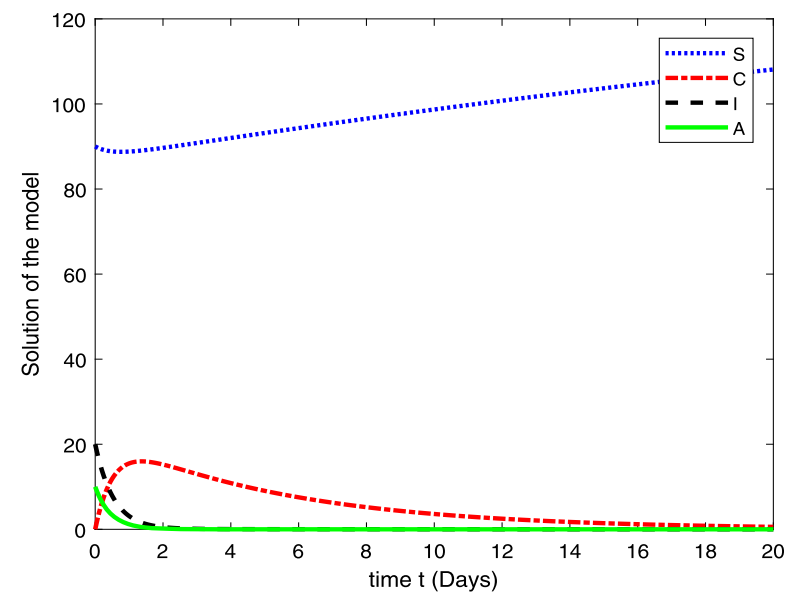

Figure 7 The solution for (1) for $\mathcal{S}, \mathcal{I}, \mathcal{C}, \mathcal{A}$, keeping $h=0.1$ and $\alpha=1$ 


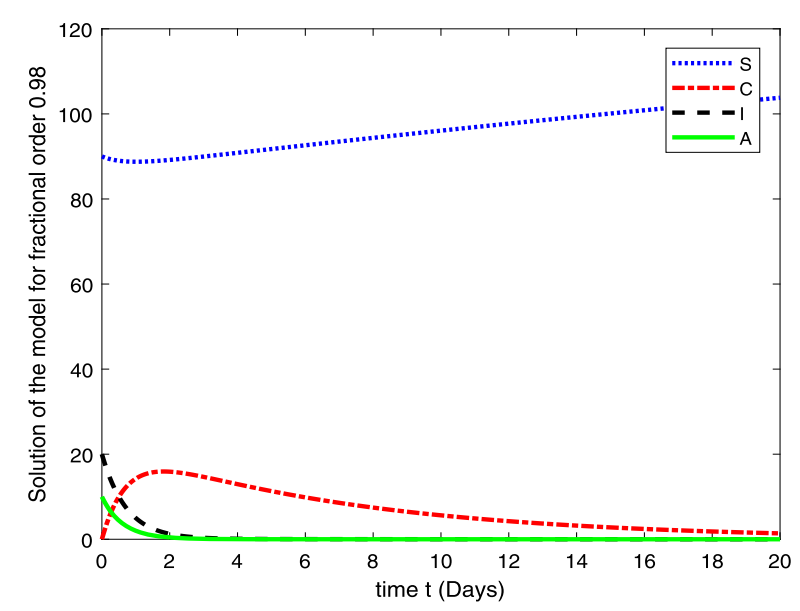

Figure 8 The solution for (1) for $\mathcal{S}, \mathcal{I}, \mathcal{C}, \mathcal{A}$, keeping $h=0.1$ and $\alpha=0.98$

ble to HIV, AIDS, and cured class. The graphs also show that our results are robust. These also illustrate asymptotic behavior around the endemic equilibrium.

\section{Conclusions}

In this paper, we considered a fractional order HIV/AIDS model in the Atangana-Baleanu sense of derivative for the existence, uniqueness of solution, Hyers-Ulam stability, and numerical simulations. The study for the existence and uniqueness of solution guaranteed that the model has a solution, while the Hyers-Ulam stability ensured its stability. These encouraged us to perform the numerical simulations of model (1). For the numerical simulations, we used the Euler approach and the given scheme. The scheme was then utilized for the numerical simulations. The joint solution of the model was given in Fig. 5, which also shows stability for $\alpha_{1}=1$, while Fig. 6 is the solution of the model for $\alpha_{1}=0.98$. We have also examined the nature of the solution by reducing the time to 20 days and observed that the solutions for the orders 1 and 0.98 are in resemblance. That is, the behavior of each class of the model is the same for fractional orders. As we get closer to the value of $\alpha_{1}$ to 1 , we get more classical results. This new model can be reconsidered for other types of fractional order derivatives, and its theoretical as well as numerical stabilities may be examined for the continuation of the study.

\section{Acknowledgements}

Thabet Abdeljawad and Aziz Khan acknowledge the support provided by Prince Sultan University for funding this work through research group Nonlinear Analysis Methods in Applied Mathematics (NAMAM) group number RG-DES-2017-01-17.

Funding

There is no source of funding for this article.

Availability of data and materials

Not applicable.

Competing interests

The authors declare that they have no competing interests. 


\section{Author details}

${ }^{1}$ Department of Mathematics, Mohi-ud-din Islamic University, Islamabad, Pakistan. ${ }^{2}$ Department of Mathematics and General Sciences, Prince Sultan University, Riyadh, Saudi Arabia. ${ }^{3}$ Department of Medical Research, China Medical University, Taichung, Taiwan. ${ }^{4}$ Department of Computer Science and Information Engineering, Asia University, Taichung, Taiwan. ${ }^{5}$ Department of Mathematics and Statistics, University of Swat, Swat, Khyber Pakhtunkhwa, Pakistan. ${ }^{6}$ Department of Mathematics, Shaheed Benazir Bhutto University, Sheringal, Dir Upper, Khyber Pakhtunkhwa, Pakistan.

${ }^{7}$ Department of Biotechnology, Shaheed Benazir Bhutto University, Sheringal, Dir Upper, Khyber Pakhtunkhwa, Pakistan.

\section{Publisher's Note}

Springer Nature remains neutral with regard to jurisdictional claims in published maps and institutional affiliations.

\section{Received: 4 October 2020 Accepted: 31 January 2021 Published online: 12 February 2021}

\section{References}

1. Sohaib, M.: Mathematical modeling and numerical simulation of HIV infection model. Res. Appl. Math. 7, 100118 (2020)

2. Perelson, S.: Modeling the Interaction of the Immune System with HIV. In: Mathematical and Statistical Approaches to AIDS Epidemiology, pp. 350-370. Springer, New York (1989). http://dl.acm.org/citation.cfm?id=90146.90450

3. Perelson, A., Kirschner, D., Boer, R.: Dynamics of HIV infection of CD4+ T-cells. Math. Biosci. 114, 81-125 (1993)

4. Nowak, M., May, R.M.: Virus Dynamics. Oxford University Press, London (2000)

5. Mukandavire, Z., Chiyaka, C., Garira, W., Musuka, G.: Mathematical analysis of a sex-structured HIV/AIDS model with a discrete time delay. Nonlinear Anal., Theory Methods Appl. 71(3-4), 1082-1093 (2009)

6. Tabassum, M.F., Saeed, M., Akgul, A., Farman, M., Chaudhry, N.A.: Treatment of HIV/AIDS epidemic model with vertical transmission by using evolutionary Pade-approximation. Chaos Solitons Fractals 134, 109686 (2020)

7. Dutta, A., Gupta, P.K.: A mathematical model for transmission dynamics of HIV/AIDS with effect of weak CD4+ T cells. Chin. J. Phys. 56(3), 1045-1056 (2018)

8. Nazir, G., Shah, K., Debbouche, A., Khan, R.A.: Study of HIV mathematical model under nonsingular kernel type derivative of fractional order. Chaos Solitons Fractals 139, 110095 (2020)

9. Sweilam, N.H., AL-Mekhlafi, S.M., Mohammed, Z.N., Baleanu, D.: Optimal control for variable order fractional HIV/AIDS and malaria mathematical models with multi-time delay. Alex. Eng. J. 59(5), 3149-3162 (2020)

10. Ma, Z., Liu, J., Li, J.: Stability analysis for differential infectivity epidemic models. Nonlinear Anal., Real World Appl. 4 $841-856(2003)$

11. McCluskey, C.C.: Lyapunov functions for tuberculosis models with fast and slow progression. Math. Biosci. Eng. 3, 513-525 (2006)

12. Khan, A., Gomez-Aguilar, J.F., Khan, T.S., Khan, H.: Stability analysis and numerical solutions of fractional order HIV/AIDS model. Chaos Solitons Fractals 122, 119-128 (2019)

13. Khan, A., Gomez-Aguilar, J.F., Abdeljawad, T., Khan, H.: Stability and numerical simulation of a fractional order plant nectar pollinator model. Alex. Eng. J. 59, 49-59 (2020)

14. Khan, A., Abdeljawad, T., Gomez-Aguilar, J.F., Khan, H.: Dynamical study of fractional order mutualism parasitism food web module. Chaos Solitons Fractals 134, 109685 (2020)

15. Khan, H., Gomez-Aguilar, J.F., Alkhazzan, A., Khan, A.: A fractional order HIV-TB coinfection model with nonsingular Mittag-Leffler law. Math. Methods Appl. Sci. 43(6), 3786-3806 (2020)

16. Atangana, A., Gomez-Aguilar, J.F.: Numerical approximation of Riemann-Liouville definition of fractional derivative: from Riemann-Liouville to Atangana-Baleanu. Numer. Methods Partial Differ. Equ. 34(5), 1502-1523 (2018)

17. Khan, M.A., Atangana, A., Alzahrani, E.: The dynamics of COVID-19 with quarantined and isolation. Adv. Differ. Equ. 2020(1), 1 (2020)

18. Atangana, A., Koca, I.: Chaos in a simple nonlinear system with Atangana-Baleanu derivatives with fractional order. Chaos Solitons Fractals 89, 447-454 (2016)

19. Owolabi, K.M., Atangana, A.: On the formulation of Adams-Bashforth scheme with Atangana-Baleanu-Caputo fractional derivative to model chaotic problems. Chaos, Interdiscip. J. Nonlinear Sci. 29(2), 1-11 (2019)

20. Abdeljawad, T., Al-Mdallal, Q.M., Jarad, F.: Fractional logistic models in the frame of fractional operators generated by conformable derivatives. Chaos Solitons Fractals 119, 94-101 (2019)

21. Abdeljawad, T., Baleanu, D.: Discrete fractional differences with nonsingular discrete Mittag-Leffler kernels. Adv. Differ. Equ. 2016(1), 232 (2016)

22. Abdeljawad, T:: A Lyapunov type inequality for fractional operators with nonsingular Mittag-Leffler kernel. J. Inequal Appl. 2017, 130 (2017). https://doi.org/10.1186/s13660-017-1400-5

23. Abdeljawad, T.: Fractional operators with generalized Mittag-Leffler kernels and their iterated differintegrals. Chaos, Interdiscip. J. Nonlinear Sci. 29(2), 023102 (2019)

24. Atangana, A., Araz, S.I.: Nonlinear equations with global differential and integral operators: existence, uniqueness with application to epidemiology. Results Phys. 20, 103593 (2020)

25. Atangana, A., Araz, S.I.: Mathematical model of COVID-19 spread in Turkey and South Africa: theory, methods and applications. medRxiv (2020) 